\title{
ESTRATEGIA GEOPOLÍTICA DE NICARAGUA SOBRE EL PROYECTO GRAN CANAL INTEROCEÁNICO DE NICARAGUA 2012
}

Dimas Antonio Delgado Alemán*

\begin{abstract}
Resumen
Para el Estado de Nicaragua la construcción de un Canal Interoceánico es una necesidad socioeconómica y cultural, coincidentemente también lo es para la comunidad internacional y el comercio marítimo mundial. Nicaragua cuenta hoy, con un marco jurídico legal que le permite celebrar convenios con inversionistas extranjeros y nacionales sosteniendo relaciones de respeto y cooperación mutua; salvaguardando los intereses nacionales. El proyecto impulsaría de manera significativa el progreso y desarrollo económico sostenible del país, protegiendo los recursos naturales y medioambientales de acuerdo a las leyes y tratados nacionales e internacionales al respecto.

Palabras clave: geopolítica, intervencionismo, soberanía nacional, proyecto canal interoceánico, imperialismo.
\end{abstract}

Key words: geopolitical situation, intervencionism, national sovereignty, inter-oceanic canal, imperialism.

\section{Desarrollo del Artículo Científico}

\section{Introducción}

\subsection{Presentación}

El anhelo de realizar un canal interoceánico en el territorio nacional ha sido un sueño de muchas generaciones de nicaragüenses, prácticamente desde el periodo colonial español.

Basta recordar que en 1524 el conquistador Hernán Cortés le enviaba carta al emperador español Carlos $\mathrm{V}$ en la cual exponía que aquel que obtuviera el paso entre los dos océanos podría considerarse el dueño del orbe.

\subsection{Objetivo}

Analizar la estrategia geopolítica de Nicaragua en el proyecto canalero interoceánico, 2012.

\subsection{Problemática y Justificación}

¿Cuál es la estrategia geopolítica de Nicaragua en el proyecto canalero interoceánico?

Esta problemática es importante porque involucra a instituciones que tienen como misión la seguridad de la población, del territorio nacional, la preservación, protección de los recursos naturales: Ejercito Nacional, Policía Nacional, MARENA. A la Cancillería de la República

\footnotetext{
* M.Sc. en Geografía. Docente del Departamento de Geografía
} 
por las relaciones de respeto y cooperación mutua con todos los países interesados en el tema. Al Instituto Nicaragüense de Estudios Territoriales (INETER) para realizar estudios topográficos y mapeos de la zona. Y a la iniciativa privada, por la incidencia en el comercio nacional e internacional que generaría el Canal. Y el desarrollo y progreso de las comunidades indígenas o étnicas localizadas en la zona de acuerdo a sus costumbres y tradiciones.

\subsection{Antecedentes}

A mediados del siglo XIX, Nicaragua da los primeros pasos para la construcción de un Canal y contrata con este objetivo en 1837-1840 a John Baily quien al concluir el estudio manifestó la perspectiva de abrir un canal interoceánico. Y en 1858 con el Tratado Cañas-Jerez, Nicaragua obtiene derechos exclusivos de canalización sobre el Río San Juan.

El apetito de grandes potencias económicas y militares como EE.UU. e Inglaterra demostró gran interés por la posición geoestratégica de Nicaragua, al intervenir directamente en las políticas internas de este país. Así en 1841, Inglaterra extendió la jurisdicción del rey misquito hasta San Juan del Norte, con el propósito deliberado de adueñarse de la ruta canalera. Posteriormente, con la firma del tratado Clayton-Bulwer EE.UU. e Inglaterra pactaron en 1850, que ninguno podía hacer el canal interoceánico de forma unilateral, obviamente sin tomar en cuenta a Nicaragua. Luego el capítulo de William Walker, quien después de declararse presidente de Nicaragua se adueñó de la ruta del tránsito (1855-1857).

EE.UU. fiel a la "Doctrina Monroe" y "El Destino Manifiesto", intervino nuevamente en Nicaragua, por causa de la construcción del canal, cuando estimó que el presidente Zelaya obstaculizaba sus intereses en el tema; por lo que usó de pretexto el caso de la muerte de Cannon y Groce para enviar el ultimátum conocido como la "Nota Knox" en 1909, lo que dio como resultado el derrocamiento del General Zelaya. Su pecado no fue estar indispuesto a construir el canal con los EE.UU. sino construirlo preservando la soberanía de Nicaragua.

Así el gobierno conservador de Adolfo Díaz, firmó un acuerdo (Tratado ChamorroBryan, 05 de agosto 1914) con el Gobierno de los Estados Unidos, otra vez por el mismo tema: la construcción de un Canal Interoceánico, ocasión en la que se le otorga, entre otras cosas, al gobierno EE.UU. una concesión exclusiva sobre los derechos precisos y convenientes para la construcción del Canal por 99 años prorrogables por otros 99 años.

Entre 1927 y 1933, en las montañas del Norte de Nicaragua, el General Augusto C. Sandino, luchaba heroicamente contra la intervención militar de los EE.UU. En este contexto manifestaba, que el progreso exigía que se construyera el Canal de Nicaragua, pero con capital de todo el mundo y no solo con capital exclusivo de los Estados Unidos.

Entre 1939 y 1948 Anastasio Somoza García, fundador de la dinastía, promovió reformas en la constitución e introdujo artículos relacionados a la firma de Tratados que tuvieran por objeto la construcción del Canal, en los cuales se concedía a Estados Unidos, el uso temporal de todo el territorio nacional, el aire, la estratosfera, las aguas litorales. Una coincidencia total de liberales y conservadores, de entonces, al servicio de los intereses imperiales.

El Dr. Arnoldo Alemán 1996-2001, creó el Acuerdo Presidencial No 436-99, "Creación de la Comisión de Trabajo para el Gran Canal Interoceánico de Nicaragua", y 
durante el periodo de gobierno del Ing. Enrique Bolaños, se dio continuidad al tema y reformó dicha comisión el 18 de marzo 2002. Como resultado de estos dos periodos, se llegó a proponer seis posibles rutas de canalización, quedando pendiente de estudio la ruta del Río San Juan.

En 2006, el presidente Bolaños anunció que el proyecto tendría un costo estimado de 18 mil millones de dólares y tomaría unos 12 años para construirse, con una extensión promedio de $280 \mathrm{Km}$., y con capacidad para buques de hasta 250.000 toneladas.

Todas las rutas se ubican en la zona suroriental de Nicaragua, partiendo de las costas del Mar Caribe entre Laguna de Perlas y el Delta del Río San Juan de Nicaragua; todas pasan por el Lago Cocibolca y el istmo de Rivas, por el sector del río Brito a desembocar en el Océano Pacífico.

\subsection{Resultados Esperados}

Que la estrategia geopolítica de Nicaragua sobre el proyecto canalero se rija a través de la Ley 800 Ley del régimen jurídico de el gran canal interoceánico de Nicaragua (03/07/2012 que plantea de manera general: a) relaciones de mutuo respeto que permitan la salvaguarda de la soberanía e independencia nacional dentro del marco delos Tratados y Convenios Internacionales y la legislación nacional, b) cooperación con los inversionistas para la bienandanza del tránsito del comercio global, continuo, eficiente, seguro y la generación de empleo. c) declararse como zona de paz, seguridad y desarrollo económico sostenible, el área de influencia del canal, previendo todas las medidas medioambientales que garanticen la protección del mismo.

\section{Materiales y Métodos}

El estudio se enmarca en el paradigma socio-crítico, porque se toma en cuenta los intereses de la nación. Procura la critica documental para identificar el potencial de Nicaragua para su cambio socioeconómico. Es de tipo documental, porque se realiza a través de la consulta de libros, periódicos, revistas, leyes y artículos en sitios Web.

Es longitudinal, porque se extiende a través del tiempo (más de cien años) dando seguimiento al fenómeno denominado proyecto canalero nicaragüense.

\section{Resultados y Discusión}

Con el presidente Daniel Ortega Saavedra sucede algo novedoso pues el proyecto canalero por primera vez tiene un marco jurídico legal, ley 800 "ley del régimen jurídico de el gran canal interoceánico de Nicaragua (03/07/2012) que le da seguridad y estabilidad a los inversionistas a través de ella y la posibilidad real de ejercer soberanía sobre dicho proyecto, con el 51\% las acciones para el Estado Nacional.

Además reglamenta la supervisión del uso racional y sostenible del medioambiente en la zona geográfica de influencia, en el marco de los convenios y tratados nacionales e internacionales. Actualmente se estudia la factibilidad de la ruta sobre el Río San Juan.

Se estima que el Canal Interoceánico podría estar concluido en 10 años a partir de la aprobación de la ley 800 y que captaría entre 416 y 573 millones toneladas métricas del 2019 al 2025. Lo que representaría el $3.9 \%$ y $4.5 \%$ de la carga marítima mundial. En la actualidad, aún con la ampliación del Canal de Panamá, la demanda del transporte marítimo no es 
compensada totalmente, ya que este canal sólo cubre, aproximadamente, un tercio de la demanda estimada para 2019 (lo que representaría un $2.9 \%$ en la carga marítima mundial).

Por tanto, un nuevo Canal Interoceánico es una necesidad mundial, y lo mejor es que el lugar más propicio para construirlo, después de tantos siglos, sigue siendo Nicaragua. Las condiciones geográficas le dan esa posibilidad, ya que posee la parte más angosta de tierra en América entre las dos masas continentales de agua, el Océano Pacífico y el Océano Atlántico y recursos naturales únicos, que incluyen una amplia disponibilidad de lagos y ríos y tierras bajas; es de menor impacto al medio ambiente, es rentable y generaría muchos inversiones y empleos. Constituyéndose en un patrimonio de la nación y por su naturaleza, tendrá las características de total neutralidad y de servicio público internacional, lo que se traduciría en el despegue económico de Nicaragua.

\section{Conclusiones y Recomendaciones}

Existe actualmente una necesidad real, a nivel nacional e internacional, de construir un Canal Interoceánico, debido al aumento de la demanda en el transporte marítimo mundial.

El lugar que reúne las condiciones óptimas para su construcción es Nicaragua, por sus recursos naturales y posición geoestratégica.

Nicaragua cuenta por primera vez con un marco Jurídico Legal Ley 800, que les da seguridad y estabilidad a los inversionistas. Y la posibilidad real de ejercer soberanía sobre dicho proyecto; con el 51\% de las acciones para el país. Reglamenta la supervisión, el uso racional y sostenible del medioambiente en la zona geográfica de influencia, en el marco de los convenios $\mathrm{y}$ tratados nacionales e internacionales.

El proyecto se convertiría en el eje principal del despegue económico de Nicaragua, priorizando las comunidades locales. Se declara como zona de paz y seguridad el área del Canal Interoceánico. Y que todas las instituciones del Estado y la sociedad en general deben aunar esfuerzos para la materialización de este proyecto de nación.

\section{BIBLIOGRAFIA}

1. Acuerdo Presidencial N0.1602012. (N.d). Extraído de www. fondacionen rique bo la ̃̃os. org AP_160_2002_03_22_ G57_REFORMA_ACUERDO_ PRESIDENCIAL_N0436-99_ CREACION_COMISION_CANAL_ INTEROCEANICO modificado 16/08/2012

2. Arellano Jorge Eduardo, (2004).La Pax Americana (1910-1932). $1^{\mathrm{a}}$.ed. Managua, fondo editorial CIRA.

3. Asamblea Nacional de la República de Nicaragua (2012). Iniciativa de Ley de Régimen Jurídico del Gran Canal de Nicaragua. Sitio Web www. asambleanacional.gob.ni [visitado 06 diciembre de 2012]

4. Canal, entre lo viable y lo inverosímil. Recuperado 08 junio de 2012 sitio Web www.elnuevodiario.com.ni

5. El Canal y Amenazas a la Soberanía. (Nd) Ministerio de Relaciones Exteriores, Nicaragua, (2006) recuperado de sitio Web www. minrex.gob.ni [visitado en agosto de 2012] 
6. Canal interoceánico sería "complementario" y no "competitivo" con el de Panamá. Recuperado 6 de junio de 2012 02:30 P.m. de www.elnuevodiario.com.ni

7. Encuesta conservadora. (n.d). Sitio Web www. fundacionenriquebolaños.org [visitado 16 agosto de 2012]

8. Canal de Nicaragua. (n.d).Extraído el 25 septiembre de 2012 de http// es.wikipedia.org/Wiki/canal_de_ Nicaragua\#historia

9. Kinloch Tijerino Frances, (2008). El primer encuentro con los filibusteros en Nicaragua: antecedentes $y$ contexto Visitado 03/09/12,11:47am http//www. afehc-historiacentroamericana. org $/$ ?action=bul_aff\&id=36

10. Kinloch Tijerino Frances Nicaragua: Identidad y Cultura Política (18211858), (1999). Banco Central de Nicaragua.

11. León Debayle. El tratado ChamorroBryan. (Nd) extraído 16 agosto de 2012 03:50 p.m de www. fundaciónenriquebolaños.org

12. Ley aprobada ayer en Managua. Recuperado 04/07/2012 12:00 PM de http://www.trincheraonline. com/2012/06/08/la-ruta-detransito-y-el-canal-interoceanicopornicaragua/

13. Ley 800 ley del régimen jurídico de el gran canal interoceánico de nicaragua y de creación de la autoridad de el gran canal interoceánico de Nicaragua. Sitio Web http//:www. asambleanacional.gob.ni/ [visitado 06 diciembre de 2012]
14. Nicaragua quiere canal interoceánico. AFP. Recuperado 08/06/2012 - 01:35 PM dehttp://www.zocalo.com.mx/ seccion/articulo/nicaragua-quierecanal-interoceanico 5

15. Nicaragua expone en Bruselas "bondades" del Canal Interoceánico. Recuperado 06 junio de 2012 sitio Web www.elnuevodiario.com.ni

16. Oquist Kelley Paul. El Canal Interocénico en el Plan Nacional de Desarrollo Humano (2012). Foro Debate "La cuenca de los Grandes Lagos y El Río San Juan: Columna vertebral. Conferencia, julio, UNANManagua (RURD).

17. Montenegro Guillén Salvador. Gran Canal Interoceánico (2012) Foro Debate “La cuenca de los Grandes Lagos y El Río San Juan: Columna vertebral. Conferencia, julio, UNAN-Managua (RURD).

18. Tratado de límites entre Nicaragua y Costa Rica. Jerez-Cañas versión digital. Sitio Web http//www. fundaciónenriquebolaños.org [visitado en agosto de 2012]

19. Taylor P.J. (1994).Geografía Política. 1 ed. Trama editorial SL. 